\title{
All-optical control and metrology of electron pulses
}

\author{
C. Kealhofer, W. Schneider, D. Ehberger, A. Ryabov, P. Baum*, F. Krausz*
}

\begin{abstract}
Affiliations:
${ }^{1}$ Ludwig-Maximilians-Universität München, Am Coulombwall 1, 85748 Garching, Germany

${ }^{2}$ Max-Planck-Institute of Quantum Optics, Hans-Kopfermann-Str. 1, 85748 Garching, Germany

*Correspondence to: ferenc.krausz@1mu.de, peter.baum@lmu.de
\end{abstract}

\begin{abstract}
Short electron pulses are central to time-resolved atomic-scale diffraction and electron microscopy, streak cameras, and free-electron lasers. We demonstrate phase-space control and characterization of 5-picometer electron pulses using few-cycle terahertz radiation, extending concepts of microwave electron pulse compression and streaking to terahertz frequencies. Optical-field control of electron pulses provides synchronism to laser pulses and offers a temporal resolution that is ultimately limited by the rise-time of the optical fields applied. We use few-cycle waveforms carried at $0.3-\mathrm{THz}$ to compress electron pulses by a factor of 12 with a timing stability of $<4$ femtoseconds (rms) and measure them by field-induced beam deflection (streaking). Scaling the concept towards multi-THz control fields holds promise for approaching the electronic time-scale in time-resolved electron diffraction and microscopy.
\end{abstract}


Electron beams controlled with temporally varying electric fields enabled the first oscilloscopes and electronic computers; today, they are essential to free-electron lasers $(1,2)$, ultrafast streak cameras (3-5) and femtosecond imaging and diffraction techniques (6-8). Microwave technology at $\mathrm{GHz}$ frequencies has been the workhorse for ultrafast electron pulse control thus far, with applications ranging from particle acceleration to ultrafast pulse compression and highresolution streak cameras. Unfortunately, microwave components suffer from appreciable phase drifts and laser-microwave synchronization below 10 fs becomes technologically challenging (911). Laser-field-driven dielectric accelerator structures operating near petahertz frequencies are being developed for next-generation particle accelerators $(12,13)$, but the short wavelength $(\sim 1$ $\mu \mathrm{m})$ and oscillation period ( $\sim 1 \mathrm{fs})$ place extreme requirements on the input beam emittance and pulse duration $(12,13)$. In between, there is the regime of $\mathrm{THz}$ frequencies. Femtosecond-lasergenerated plasma waves exhibit THz longitudinal fields, which can accelerate electrons efficiently to the relativistic and ultrarelativistic energy domain (14). However, the excessive energy, divergence and insufficient timing control of the resultant several-to-multi-MeV electron pulses impair their utility for most of the above applications and particularly for ultrafast electron diffraction and microscopy.

Pulsed THz fields can be generated in a much more controlled way from the coherent nonlinear polarization of matter, e.g. by optical rectification (15). The pulses can reach field strengths of the order of $10^{10} \mathrm{~V} / \mathrm{m}(16)$ and their half-cycle durations ideally match practical electron bunch dimensions (fs-ps in time and $\mu \mathrm{m}-\mathrm{mm}$ in diameter). In addition, THz control fields derived from a single ultrafast laser via nonlinear optics result in near-perfect (potentially sub-femtosecond) temporal synchronization, offering the potential to substantially surpass the performance of microwave-laser synchronization and obviating the need for locking electronics. Therefore, $\mathrm{THz}$ and infrared radiation generated from laser-driven coherent nonlinear polarization is ideally suited for controlling electron pulses, as indicated by simulations (17-21) and recent experiments on controlling nanoscale photoemission (22) and electron acceleration in a dielectric waveguide (23).

\section{Concept and implementation}

Figure 1 depicts the experimental implementation of our THz-field-controlled electron beamline consisting of two functional units, one for pulse compression and one for temporal characterization by streaking (24). Near-1-ps pulses from a Yb:YAG-based regenerative 
amplifier (25) drive two optical rectification stages for generating few-cycle THz pulses. With the same laser, electron pulses with on average $\sim 1$ electron per pulse are generated by twophoton photoemission from a gold thin-film photocathode and electrostatically accelerated to 70 $\mathrm{keV}$. The optical rectification stages produce near-single-cycle pulses at a carrier frequency of $0.3 \mathrm{THz}$ with pulse energies up to $40 \mathrm{~nJ}$, implying field strengths of up to $\sim 10^{6} \mathrm{~V} / \mathrm{m}$ under gentle focusing conditions. These are used to control the electron pulse's momentum, energy, duration or timing.

We use butterfly-shaped metal resonators (inset to Figure 1) to mediate the interaction between the electrons and the terahertz fields. The THz electric field is enhanced in the plane of the resonators and confined to sub-wavelength dimensions so that energy and momentum conservation in the electron-photon interaction can be satisfied (26).

Electrons passing through the resonator structures experience a net change in momentum that corresponds to the integral of the Lorentz force along their trajectories. This change varies periodically as a function of arrival time of the electron in the $\mathrm{THz}$ field. The first $\mathrm{THz}$ control stage uses a tilted resonator to provide force components longitudinal to the electron beam for temporal compression. The second $\mathrm{THz}$ control stage, oriented normal to the beam, provides a delay-dependent deflection for temporal characterization.

The root-mean-square ( $\mathrm{rms}$ ) electron beam radius is $3 \mu \mathrm{m}$ at the compression resonator and $11 \mu \mathrm{m}$ at the streaking resonator, at least 8 times smaller than the resonator mode dimensions. Because the electron pulse from the source is shorter than $1 \mathrm{ps}$ and hence shorter than the half cycle of the $\mathrm{THz}$ field, the latter exerts a uniform but time-dependent force on the electron wavepacket in all dimensions. This force imparts acceleration and/or compression at the first stage and time-dependent deflection (i.e. streaking) at the second stage. As a result, the pulsed electron beam is entirely under control of a single intense-pulse laser system and its optical fields.

\section{Electron pulse compression}

Even without any space charge, electron pulses unavoidably have a finite duration after acceleration due to dispersion (27). Electron pulse compression is therefore indispensable for reaching sub-phonon or electronic timescales in diffraction (28). Electron pulse compression requires deceleration and acceleration of the electron when arriving before or after the mean electron arrival time, respectively. For pulse compression with $\mathrm{THz}$ fields, the first butterfly 
microstructure is oriented at an angle of $45^{\circ}$ with respect to the electron beam. THz pulses are incident at an angle of $45^{\circ}$ at the metal surface and hence normal to the electron beam (Fig. 1), so that the THz-excited resonance provides time-dependent force components parallel to the electron beam. In analogy to microwave compression, the time-dependent fields permit compression of the electron pulse substantially below its initial duration (28) and hence down to attosecond duration in the absence of space charge (27). Note that in the limit of one or fewelectron pulses, the pulse duration is understood as the ensemble distribution of electron arrival times with respect to the laser reference.

The effective strength of the compression stage is quantified by $g_{E}$, the energy imparted to the electrons in the forward direction with changing delay time. In the experiment, $g_{E}$ is proportional to the incoming $\mathrm{THz}$ peak field strength divided by the cycle period. After the interaction, the electron pulse becomes shorter as it propagates, reaching a minimum duration at a distance

$$
f_{c}=m_{e}(\gamma \beta c)^{3} / g_{E}
$$

where $\mathbf{c}$ is the speed of light, $\mathbf{m}_{\mathbf{e}}$ is the mass of the electron, $\boldsymbol{\beta}$ is the ratio of the electron speed to the speed of light, and $\boldsymbol{\gamma}=\mathbf{1} / \sqrt{ }\left(\mathbf{1}-\boldsymbol{\beta}^{2}\right)$. Localized THz fields enhanced to merely $10^{6} \mathrm{~V} / \mathrm{m}$ are sufficient to yield a $\mathbf{g}_{\mathrm{E}}$ of $50 \mathrm{eV} / \mathrm{ps}$ and hence produce a convenient temporal focus at a distance of tens of centimeters from the compression stage, depending on central energy (24).

\section{Temporal characterization}

To measure the temporal profile of the electron pulse, the second stage is configured to provide a terahertz-driven delay-dependent deflection, which we dub streaking, in analogy to microwavedriven and laser-field-driven devices. We first tested the temporal characterization with the uncompressed pulse. The time-dependent deflection of the beam yields a deflectogram (Fig. 2B), i.e. the beam profile (in the plane of deflection) plotted vs. delay of the electron pulse with respect to the THz field. At the zero crossings of the field, the deflection is a steep function of time, and the beam width increases due to the finite-duration electron pulse. Raw images of the electron beam were recorded on the phosphor-covered camera with the $\mathrm{THz}$ deflecting field switched on and off and timed to yield maximum streaking of the beam (Fig. 2A). At the maxima and minima, minimal spreading occurs. The electron pulse duration and the timedependent optical forces are determined by fitting the data of Fig. 2B assuming an instantaneous 
momentum transfer transverse to the electron beam induced by the THz field as a function of the electron arrival time.

The deflection is proportional to the integral of the Lorentz force along the electron's trajectory and closely follows the electric field's temporal profile inside the slit due to near field confinement, as a consequence of the electron traversing the field-enhanced region within a tiny fraction of the field oscillation period. This is in sharp contrast with attosecond streaking, which operates in the opposite limit (transit time much longer than the streaking field duration) so that the streaking spectrogram mimics the vector potential of the streaking field (29). A spline interpolation through four support points per picosecond is used for modelling time-dependent deflection, and a Gaussian temporal profile is assumed for the electron pulse. Figure 2C shows the calculated deflectogram with the fitted time-dependent deflection. The electron pulse duration is $930 \mathrm{fs}$ full width at half maximum (FWHM), consistent with a propagation-induced temporal spread resulting from a $0.6-\mathrm{eV}$ initial energy spread of the electron pulse.

The deflectogram displays sustained deflection oscillations far beyond the duration of the near-single-cycle incident $\mathrm{THz}$ driving pulse. The best fit yields a resonance frequency of $0.29 \pm 0.01 \mathrm{THz}$ of the resonator with a decay rate of $4.5 \pm 0.4$ ps resulting from radiative and resistive damping.

Figure 2D shows the resultant maximum streaking speed as a function of incident peak THz field strength in this range. The highest value measured exceeds $8 \mu \mathrm{rad} / \mathrm{fs}$, corresponding to a streaking speed of $4.4 \mu \mathrm{m} / \mathrm{fs}$ at the camera in our experimental geometry. The distance over which all momentum change accumulates is determined by the THz near-field decay length $(\sim 100 \mu \mathrm{m})$ and the foil thickness $(\sim 30 \mu \mathrm{m})$. The resulting interaction length is some 100 times smaller than in conventional streak cameras $(3,4)$, removing a significant limitation of their temporal resolution.

In the THz-field-driven streak camera, temporal resolution is dictated by the beam quality and signal-to-noise ratio. At an incident $\mathrm{THz}$ field strength of $1.3 \times 10^{6} \mathrm{~V} / \mathrm{m}$, the root mean square (rms) beam width on the camera of 1.6 pixels $(25 \mu \mathrm{m})$ corresponds to 6-fs resolution (rms). With $\sim 1000$ detected electrons, the beam quality and signal-to-noise allow detection of displacements or spot width changes of 0.1 pixels $(1.56 \mu \mathrm{m})$. A displacement of 0.1 pixels corresponds to $0.4 \mathrm{fs}$ accuracy of arrival time measurements, whereas a 0.1 pixel increase of the spot size from the unstreaked value corresponds to a 2 fs (rms) or $\sim 4.5$ fs (FWHM) pulse duration. These 
resolutions are achieved with a signal accumulation time of $6 \mathrm{~s}$ and improve with longer integration. These values represent a near-two-order-of-magnitude improvement over the stateof-the-art of microwave streak cameras $(3,4)$, obtained already at THz fields of $\sim 10^{6} \mathrm{~V} / \mathrm{m}$ as opposed to $10^{10} \mathrm{~V} / \mathrm{m}$ available (16). As a consequence, our THz streaking concept may allow the direct measurement of sub-femtosecond electron pulses $(30,31)$ and their timing drifts.

\section{Compression and measurement}

Figure 3A and 3B show streaking deflectograms with and without THz-field compression, respectively. In the latter, the $\mathrm{THz}$ field strength of the compression stage has been adjusted to produce a temporal focus at the streaking stage. A pronounced sharpening of the trace can be seen in the vertical (streaking) direction, indicating a substantial reduction in electron pulse duration.

Figure 3C shows the systematic evolution of measured electron temporal profile for a varying compression strength $g_{E}(32)$. The deflection as a function of time is reconstructed and used to perform the nonlinear transformation between the spatial profile of the streaked pulse and the underlying temporal profile of the THz-field-manipulated incident pulse (24). The dotted lines depict the results of three-dimensional electron trajectory simulations (32).

The electron pulse (blue) first shortens to a minimum duration ( $5^{\text {th }}$ trace) and afterwards lengthens again, with a double-peaked shape that is characteristic of over-compression by a sinusoidal field (30). Figure 3D shows the experimental and theoretical pulse durations versus THz-field strength and average power of the driving laser. The shortest pulse (inset to Fig. 3D) has a FWHM duration of $75 \mathrm{fs}$, in excellent agreement with the simulation and a factor of 12 shorter than the original, 930-fs pulses and shorter than the half period of many fundamental phonon modes and molecular vibrations. Comparison of the shortest measured pulse shape with a Gaussian profile (dotted line) reveals a deviation; this is a consequence of the THz field's residual curvature over the time scale of the incoming, uncompressed electron pulse. Optimized electron sources with sub-100-fs duration (33) will reduce this effect; the particle tracing simulations (24) show that 3-fs (FWHM) compressed pulses can be generated with a smaller source size and the 100-fs incoming pulses achievable if the photoemission energy is matched to the work function $(7,27)$. This would be an order of magnitude shorter than the 28-fs (FWHM) pulses generated in the single-electron regime so far (28) and allow the study of light-driven electronic motion via sub-cycle diffraction $(34,35)$. 


\section{Electron-laser timing metrology}

Femtosecond pump-probe crystallography with electrons (7) or with x-ray free-electron lasers ( 1 , 2) suffers from laser-electron timing jitter at the sample location, typically caused by imperfect laser-microwave synchronization $(10,11)$. In contrast to the statistical electronic processes in the photodiodes used for microwave synchronization (9), laser-generated $\mathrm{THz}$ fields are, within the attosecond response time of the underlying nonlinear polarization, perfectly locked in time to the intensity profile of the pump pulses. Timing jitter/drift between the laser pump pulse and the electron probe pulse can only originate from extrinsic effects, such as fluctuations in path lengths or laser pulse energy.

Experimentally, we studied drifts of the electron pulse with respect to the $\mathrm{THz}$ field by setting the near-field-enhanced streak camera to a constant delay and recording the beam deflection over time (Fig. 4A). First, we scrutinized a possible role of amplitude-to-timing conversion in the nonlinear optical rectification process. Figure 4B shows the change in electronTHz-field timing when varying the laser pump pulse intensity before the THz generation crystal. We observe a systematic change in timing, but the slope at the operation conditions $(7 \mathrm{~W})$ is only $1.0 \mathrm{fs}$ per one percent change in laser power, which is negligible for our laser system, whose intensity drifts and shot-to-shot energy fluctuations are below 2\% (25).

In a second experiment, we measured drifts of the photoemission electrons with respect to the streaking THz field by turning the THz control field off. For periods longer than $1 \mathrm{~s}$ we obtain 4.6 fs (rms) over 15 minutes (Fig. 4C). Finally, we repeated this measurement with the THz-driven pulse compression activated, with integration times of $6 \mathrm{~s}$ and after $\sim 2 \mathrm{~h}$ of laboratory thermalization, measuring $3.7 \mathrm{fs}$ rms over three hours (Fig. 4E). This vastly outperforms the long-term stability of state-of-the-art laser-microwave synchronization with feedback loops (7) and also compares favorably to the record value (5 fs rms) achieved with direct microwave extraction from a laser pulse train and data post-processing (9). We expect that the measured, residual 3.7-fs drift could be further decreased by minimizing the (currently meterscale) optical path lengths and/or stabilizing them interferometrically. We note that synchronization in the all-optical scheme is entirely single-pulse-based and therefore fewfemtosecond stability can be maintained at any — and especially at very low-laser repetition rate. A clock or master oscillator is not required. 


\section{Direct THz-electron interaction at a foil: a THz cathode-ray-tube oscilloscope}

Enhancement of the $\mathrm{THz}$ field by a microstructure resonator has proven highly beneficial for electron pulse control and metrology, but some experiments might require more direct control, avoiding the localization and limited spectral response inextricably linked to resonators. For a direct electron- $\mathrm{THz}$ interaction, we realized a resonator-free interaction geometry based on a symmetry-breaking surface $(36,37)$. In the depicted concept (Fig. 5A), a thin metal foil (70-nm aluminum) acts as a mirror for $\mathrm{THz}$ radiation, and electrons transmitted through it experience an abrupt extinction of the electromagnetic field leading to net deflection. The combination of angles is chosen such that extended beam profiles experience a homogeneous time-dependence owing to lateral phase matching (37).

Figures 5C and 5D depict measured and calculated streaking deflectograms of an uncompressed electron pulse at an energy of $90 \mathrm{keV}$, exhibiting excellent agreement. Although the peak deflection is smaller than for the resonator-based setting, the deflectogram clearly exhibits single-cycle behavior, preserving the incident THz pulse's ultrabroadband spectrum without alteration (25). Figure 5B shows the peak-to-peak deflection as a function of the incoming THz field strength, revealing the expected linear dependence. Electrons delivered in a nearly collimated beam are directly (and spatially uniformly) deflected directly with the field cycles, advancing the century-old cathode-ray-tube oscilloscope to the terahertz domain. Owing to the 1-fs-scale timing accuracy of the sampling electron pulse, the cut-off frequency of this $\mathrm{THz}$ cathode-ray oscilloscope is dictated by the inverse electron pulse duration, $>10 \mathrm{THz}$ with the demonstrated compressed pulses, $>100 \mathrm{THz}$ with the few-fs electron pulses predicted by the simulations, and $>\mathrm{PHz}$ with the isolated attosecond electron pulses feasible with multi-stage compression (see below).

\section{Conclusions and outlook}

Our demonstrated generic and scalable all-optical methodology for the control and characterization of ultrafast electron pulses. used laser-generated sub-cycle $\mathrm{THz}$ transients for twelve-fold electron pulse compression, followed by a temporal profile characterization with a 10 -fs resolution. Electron-transparent foils mediating the electron-field interaction permit timeenergy phase-space manipulation of collimated electron beams of any size and, conversely, the temporal characterization of optical field transients up to frequencies only limited by the electron 
pulse duration, which - as opposed to the photon pulse in electro-optical sampling - may be shortened to less than 1 fs (27). Alternatively, resonant structures dramatically lower the $\mathrm{THz}$ power by a factor $>1000$ for effective compression and characterization of ultrashort electron pulses. Whereas the former opens the prospect of a PHz-bandwidth cathode-ray oscilloscope as an alternative to attosecond photon-pulse metrology (29), the latter may lead to unprecedented spatio-temporal resolutions in pump-probe electron diffraction and imaging.

The moderate field strengths applied in our experiment offer the potential for increasing the interaction strengths by more than two orders of magnitude, limited by the high values of electrical breakdown at $\mathrm{THz}$ frequencies. This will result in correspondingly reduced propagation distances between subsequent THz control stages, which, in turn, should improve the passive few-fs timing stability into the sub-femtosecond regime. Simultaneous compression of 100 -fsscale input electron pulses to near-1-fs duration will permit spatiotemporal imaging of the fastest structural and infrared-field-driven electron dynamics in molecular systems (35) as well as condensed matter (34) by single-electron diffraction (38) or microscopy (6) and allow measuring microscopic/atomic scale electric field waveforms up to frequencies of visible light.

The demonstrated all-optical control may also be helpful for manipulating ultra-bright multi-electron bunches. Compact electron guns have demonstrated sub-200 fs electron pulses with sufficient charge for single-shot structural dynamics studies (7). Our concept is directly applicable to them as well. Alternatively, single-shot ultrafast electron diffraction may be advanced into the few-femtosecond regime and possibly beyond by THz-streaking 100 -fs-scale probe pulses after passage through the sample (4). The unprecedented temporal resolution of the THz-driven streak camera could also be used to characterize electron micro-bunching in freeelectron lasers, supplementing THz-based diagnostics for the X-ray output $(39,40)$. The demonstrated concept is scalable to higher $\mathrm{THz}$ frequencies and multiple stages, offering the potential for cascaded compression into the sub-femtosecond regime or direct injection into a single optical cycle of a laser-field accelerator (12). This may, in the long run, lead to isolated attosecond electron pulses for recording dynamic changes of electron distribution in complex systems, including biological molecules and solid-state nanostructures. 


\section{References and Notes}

(1) W. Ackermann et al., Nature Photonics 1, 336 (2007).

(2) P. Emma et al., Nature Photonics 4, 641 (2010).

(3) G. H. Kassier et al., Review of Scientific Instruments 81, 105103 (2010).

(4) C. M. Scoby, R. K. Li, E. Threlkeld, H. To, P. Musumeci, Applied Physics Letters 102, $023506(2013)$.

(5) C. Kealhofer et al., Optics Letters 40, 260 (2015).

(6) D. J. Flannigan, A. H. Zewail, Accounts of Chemical Research 45, 1828 (2012).

(7) G. Sciaini, R. J. D. Miller, Reports On Progress In Physics 74, 096101 (2011).

(8) J. Tenboer et al., Science 346, 1242 (2014).

(9) M. Walbran, A. Gliserin, K. Jung, J. Kim, P. Baum, Physical Review Applied 4, 044013 (2015).

(10) S. Schulz et al., Nature Communications 6, 5938 (2015).

(11) G. J. H. Brussaard et al., Applied Physics Letters 103, 141105 (2013).

(12) R. J. England et al., Reviews of Modern Physics 86, 1337 (2014).

(13) E. Hemsing, G. Stupakov, D. Xiang, A. Zholents, Reviews of Modern Physics 86, 897 (2014).

(14) E. Esarey, C. B. Schroeder, W. P. Leemans, Reviews of Modern Physics 81, 1229 (2009).

(15) M. C. Hoffmann, J. A. Fülöp, Journal of Physics D: Applied Physics 44, 083001 (2011).

(16) M. Shalaby, C. P. Hauri, Nature Communications 6, 5976 (2015).

(17) S. D. Vartak, N. M. Lawandy, Optics Communications 120, 184 (1995).

(18) R. B. Yoder, J. B. Rosenzweig, Physical Review Special Topics-Accelerators and Beams 8, 111301 (2005).

(19) L. J. Wong, A. Fallahi, F. X. Kaertner, Optics Express 21, 9792 (2013).

(20) J. Fabianska, G. Kassier, T. Feurer, Scientific Reports 4, 5645 (2014). 
(21) S. R. Greig, A. Y. Elezzabi, Applied Physics Letters 105, 041115 (2014).

(22) L. Wimmer et al., Nature Physics 10, 432 (2014).

(23) E. A. Nanni et al., Nature communications 6, 8486 (2015).

(24) Materials and methods are available as supplemental materials on Science online .

(25) W. Schneider et al., Optics Letters 39, 6604 (2014).

(26) F. J. G. de Abajo, M. Kociak, New Journal of Physics 10, 073035 (2008).

(27) E. Fill, L. Veisz, A. Apolonski, F. Krausz, New Journal of Physics 8, 272 (2006).

(28) A. Gliserin, M. Walbran, F. Krausz, P. Baum, Nature Communications 6, 8723 (2015).

(29) R. Kienberger et al., Nature 427, 817 (2004).

(30) P. Baum, A. H. Zewail, Proceedings of the National Academy of Sciences of the United States of America 104, 18409 (2007).

(31) A. Feist et al., Nature 521, 200 (2015).

(32) General Particle Tracer code (http://www.pulsar.nl/gpt) .

(33) J. Hoffrogge et al., Journal of Applied Physics 115, 094506 (2014).

(34) V. S. Yakovlev, M. I. Stockman, F. Krausz, P. Baum, Scientific Reports 5, 14581 (2015).

(35) H. C. Shao, A. F. Starace, Physical Review Letters 105, 263201 (2010).

(36) T. Plettner et al., Physical Review Letters 95, 134801 (2005).

(37) F. O. Kirchner, A. Gliserin, F. Krausz, P. Baum, Nature Photonics 8, 52 (2014).

(38) S. Lahme, C. Kealhofer, F. Krausz, P. Baum, Structural Dynamics 1, 034303 (2014).

(39) U. Fruehling et al., Nature Photonics 3, 523 (2009).

(40) I. Grguras et al., Nature Photonics 6, 851 (2012).

(41) M. Aidelsburger, F. O. Kirchner, F. Krausz, P. Baum, Proceedings of the National Academy of Sciences of the United States of America 107, 19714 (2010).

(42) M. Theuer et al., Applied Physics Letters 88071122 (2006).

(43) D. Kreier, D. Sabonis, P. Baum, Journal of Optics 16, 075201 (2014). 
Acknowledgments: This work was supported by the European Research Council and the Munich-Centre for Advanced Photonics. We thank Dagmar Frischke for preparing ultrathin aluminum foils. The authors declare no competing financial interests. 
Fig. 1 Experimental setup. A 1-ps laser pulse from a $Y b: Y A G$ regenerative amplifier is frequency doubled and generates electron pulses from a thin-film gold photocathode. The laser also drives two optical-rectification stages, each generating single-cycle $\mathrm{THz}$ pulses with energy of up to $40 \mathrm{~nJ}$. THz resonator structures are laser-machined in a 30$\mu \mathrm{m}$-thick aluminum foil (inset). A first element, used for compression, is oriented at $45^{\circ}$ to the electron beam, providing time-dependent longitudinal forces on the electrons. The second $\mathrm{THz}$ resonator, used for streaking, is oriented normal to the beam, resulting in time-dependent transverse deflection.

Fig. 2 All-optical THz streak camera. (A) Images of the electron beam on the camera with and without the THz field. The full width at half maximum of the streaked beam is $\sim 50$ times larger than that of the unstreaked spot. (B) Time-dependent deflection (deflectogram) measured by varying the delay between the electron pulse and the streaking THz. Images of the beam are integrated along the unstreaked direction to determine a one-dimensional profile at each time-delay. (C) Simulated deflectogram, result of a fit simultaneously characterizing the electron pulse duration and the streaking field, dotted line. The fitted electron pulse duration is 930 fs. (d) Streaking speed vs. input $\mathrm{THz}$ field strength; the linear relationship supports a direct, field-driven interaction.

Fig. 3 THz-driven electron pulse compression. Comparison of deflectograms for (A) uncompressed (930 fs) and (B) compressed (75 fs) pulses at time-steps of $200 \mathrm{fs}$ and 10 fs, respectively. The deflectograms were measured sequentially and have a temporal resolution of $\sim 9 \mathrm{fs}$ (rms). (C) Measured temporal profiles of the electron pulses (blue traces) as the compression strength is increased (black arrow), taking into account for long pulses the curvature of the time-dependent deflection. Simulated temporal profiles (10 4 particle trajectories) (24) are superimposed (dotted black lines). Differences between the measured and simulated pulses are due in part to the curvature of the streaking field, which reduces the streaking time-resolution at times far from zero. (D) Electron pulse duration (FWHM) vs. incident THz field strength. The measured values 
are plotted as blue diamonds, and the simulation is shown as a dotted black line. The inset shows the shortest measured pulse profile $\left(5^{\text {th }}\right.$ trace from the bottom of part (C)).

Fig. 4 Passive few-femtosecond synchronization. (A) Concept for measuring arrival-time drifts between the uncompressed electron pulses and the optical streaking field. (B) Systematic coupling of laser fluctuations to timing drifts. Measuring shifts in the $\mathrm{THz}$ zero-crossing time with increasing laser pump power reveals a slope of less than $1 \mathrm{fs}$ per $1 \%$ change in laser power in a wide range of operation conditions. (C) Measured timing drift between photoemitted electron pulses and the $\mathrm{THz}$ field cycles at $10 \mathrm{~s}$ intervals. The integration time for each data point is $1 \mathrm{~s}$. (D) Concept for measuring the arrivaltime drift between THz-compressed electron pulses and the streaking field cycles. (E) Result with 6-s integration times reveals passive longer-term synchronization. The jitter values in (C) and (E) are rms deviations; statistical errors are $3.5 \mathrm{fs}$ and $2.7 \mathrm{fs}$, respectively.

Fig. 5 Resonance-free single-cycle streaking. (A) Resonator-free concept for mediating the electron- $\mathrm{THz}$ interaction using an ultrathin $\mathrm{THz}$ mirror. The angles of the $\mathrm{THz}$ beam and the foil are chosen for lateral phase matching across arbitrarily large electron and $\mathrm{THz}$ beam profiles. (B) Measurement of peak deflection vs. incident $\mathrm{THz}$ field strength. (C) Experimental deflectogram revealing single-cycle behavior. (D) Simulated deflectogram with an electron pulse duration of $800 \mathrm{fs}$. 


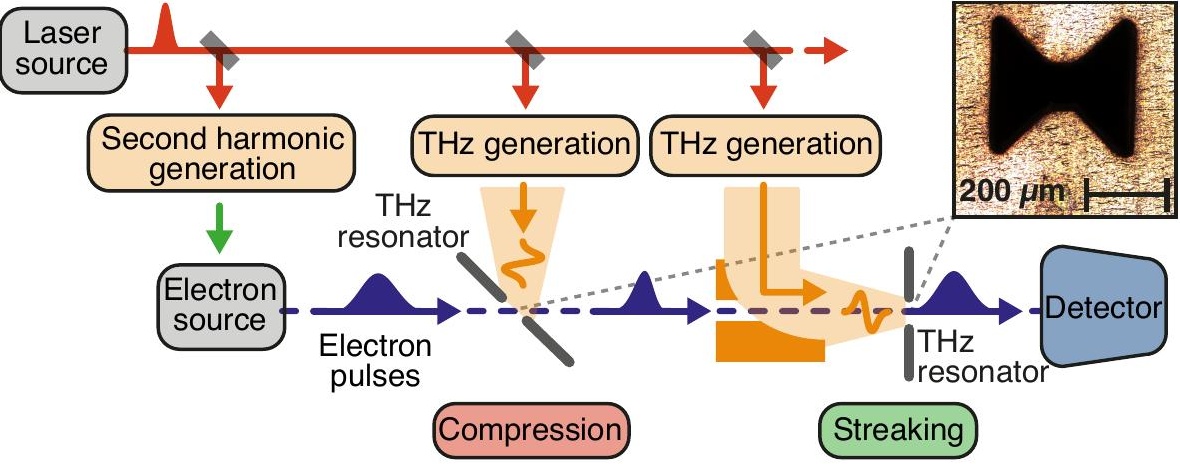




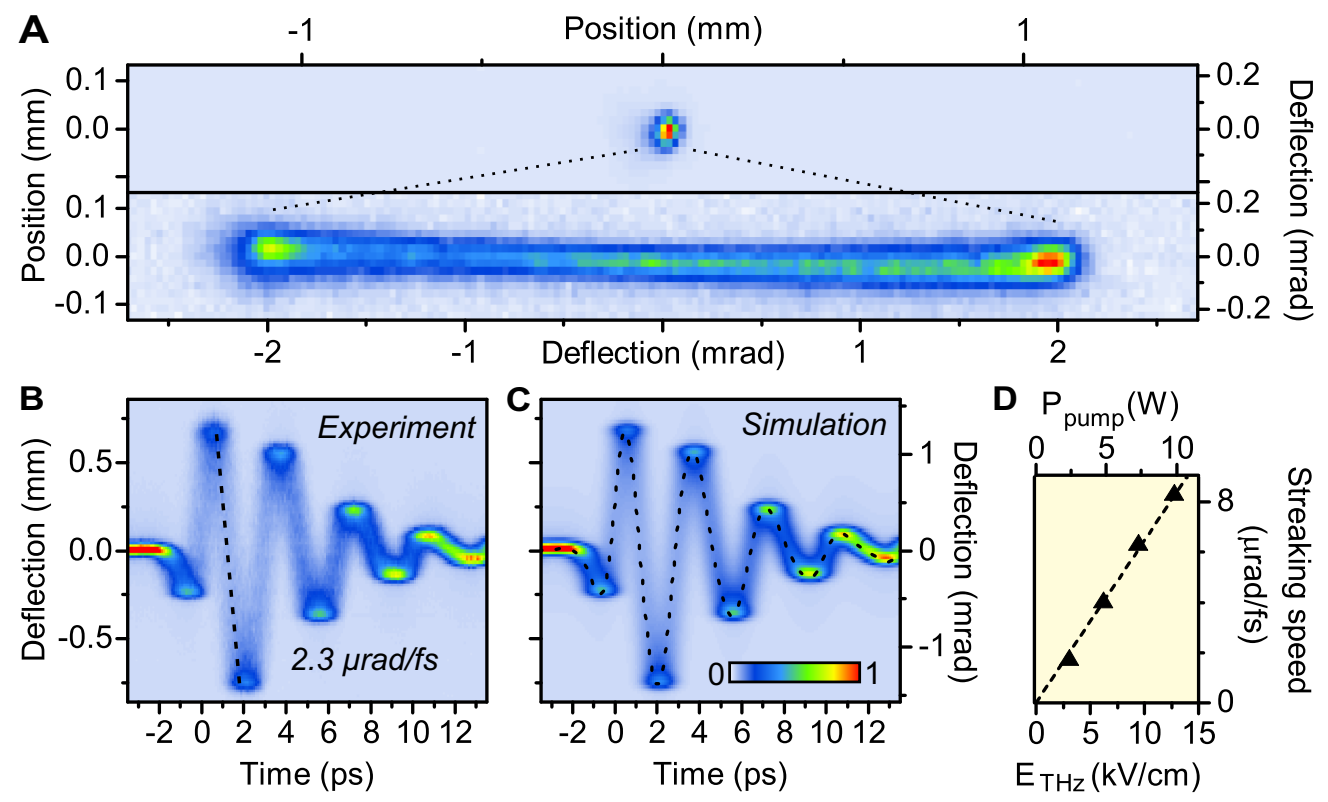



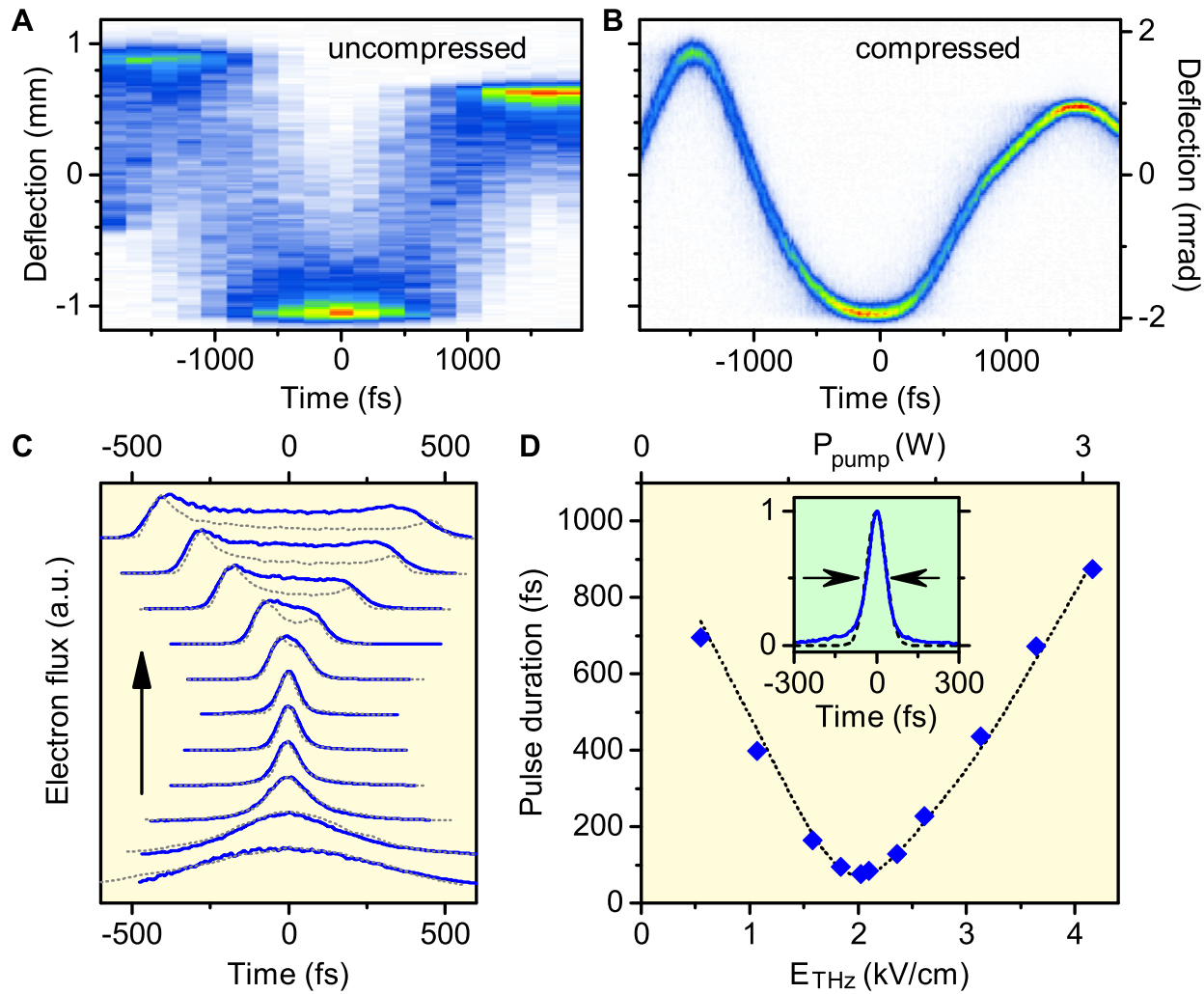


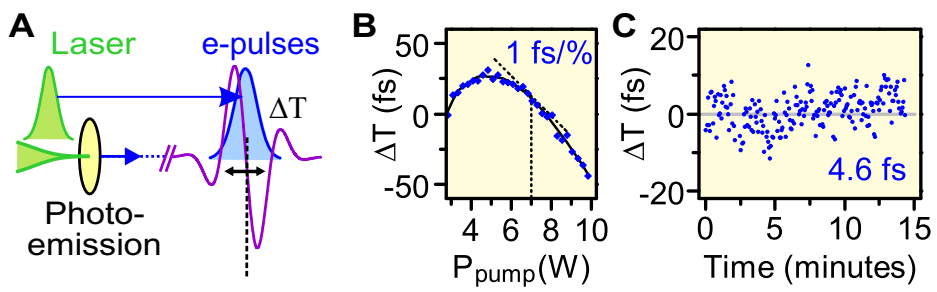

D

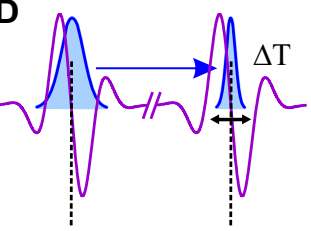

Compression

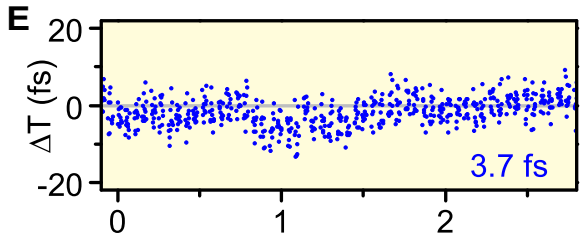

Time (hours) 
A

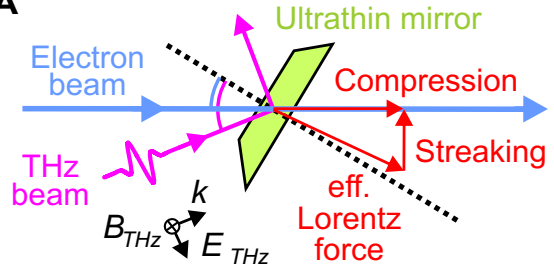

C

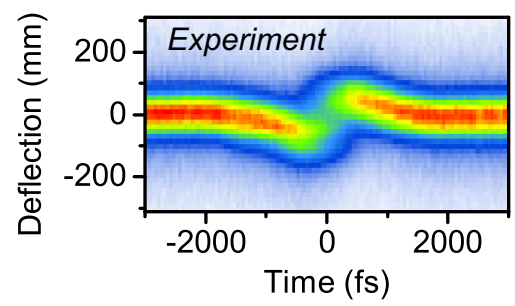

B

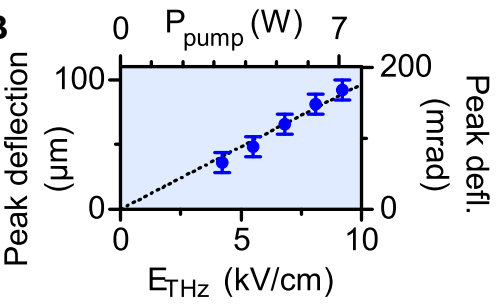

D

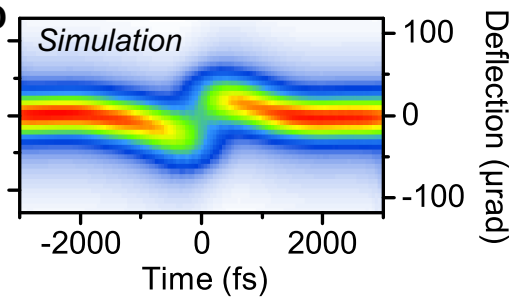




\section{Materials and Methods}

Experimental setup

The laser system is a Yb:YAG regenerative amplifier (25) which generates $1 \mathrm{ps,}$ $\sim 350 \mu \mathrm{J}$ pulses with a central wavelength of $1030 \mathrm{~nm}$ and at a repetition rate of $50 \mathrm{kHz}$. A small fraction of the laser output power is frequency doubled to obtain pulses at 515 $\mathrm{nm}$ for photocathode excitation. The cathode consists of a $20 \mathrm{~nm}$ gold film on a sapphire substrate, which is illuminated from behind. Electrons are emitted via two-photon photoemission. $<100 \mu \mathrm{W}$ are used for photocathode excitation, the electron source size is $\sim 5 \mu \mathrm{m}$ rms (fitting the spot size on the camera as a function of magnetic lens parameters). The electron gun has a cathode-anode spacing of $25 \mathrm{~mm}$, resulting in fields of $3.6 \mathrm{MV} / \mathrm{m}$ and $2.8 \mathrm{MV} / \mathrm{m}$ at $90 \mathrm{keV}$ and $70 \mathrm{keV}$ operating voltages, respectively. From the measured, uncompressed pulse durations of $780 \mathrm{fs}$ at $90 \mathrm{keV}$ and $930 \mathrm{fs}$ at $70 \mathrm{keV}$ and assuming an initial pulse duration of $\sim 500 \mathrm{fs}$ (from the two consecutive two-photon processes), we infer a longitudinal energy spread of $0.6 \mathrm{eV}$ on emission. This is consistent with the measured $4.26 \mathrm{eV}$ work function of similar thin-film gold cathodes (41).

Terahertz fields were generated by optical rectification in lithium niobate; at $7 \mathrm{~W}$ pump power, both generation stages produce $\sim 30-40 \mathrm{~nJ}$ single-cycle pulses at a frequency of $\sim 0.3 \mathrm{THz}$. The streaking terahertz was generated using tilted-pulse-front pumping (15) (for details of the experimental setup, see (25)). For streaking with the butterfly aperture, the $\mathrm{THz}$ was focused from inside the vacuum chamber and collinearly with the electron beam, using an off-axis parabolic mirror with a central hole of $\sim 3 \mathrm{~mm}$ diameter.

The compression terahertz was generated using a Cerenkov scheme (42). This approach is more compact and less alignment sensitive than the tilted pulse-front method, which is advantageous here because a delay line in the pump beam was used to control the time-delay of this terahertz field. The compression terahertz was focused with an offaxis parabolic mirror onto the butterfly antenna from outside the vacuum chamber through a $6 \mathrm{~mm}$ thick silicon window, with an effective focal distance of about $\sim 100 \mathrm{~mm}$.

In addition to the delay stage in the compression $\mathrm{THz}$ generation path, there is a delay stage in the $515 \mathrm{~nm}$ beam, before electron generation. Data collection and the control of the delay stages are automated, and the delay stages can be moved together (for example, to change the time-delay of the compressed pulses with respect to the streaking $\mathrm{THz}$.

The distance between the compression interaction and the streaking interaction is $\sim 0.49 \mathrm{~m}$. The detector (TemCam-F416, TVIPS GmbH) is located $\sim 0.55 \mathrm{~m}$ further after the streaking. A first solenoid lens focuses the beam to a spot size of $3 \mu \mathrm{m}$ (rms) in the compression aperture, and a second lens focuses the beam between the streaking aperture and the camera. The lenses are mounted kinematically to allow precise alignment and avoid temporal distortions (43), and deflection coils are used to fine-tune the electron alignment at the streaking aperture. A $50-\mu \mathrm{m}$-diameter aperture placed $\sim 100 \mathrm{~mm}$ before the butterfly resonator is used to improve the transverse beam emittance. The focus of the beam is placed between the butterfly resonator and the camera, resulting in spot sizes of $11 \mu \mathrm{m}(\mathrm{rms})$ at the resonator and $23 \mu \mathrm{m}(\mathrm{rms})$ at the camera. Beam sizes in the compression and streaking apertures were determined using knife-edge scans. The same electron optical configuration was used regardless of whether compression was applied. 
The butterfly resonators are laser-machined in 30- $\mu \mathrm{m}$-thick aluminum foil. They were designed for a resonance frequency of $0.3 \mathrm{THz}$, and simulations predict field enhancement by a factor of $\sim 5$ in the center of the resonator. Simulations of the mode profile show that even for the $11 \mu \mathrm{m}$ spot size at the streaking resonator, the amplitude of the deflection varies by less than $1 \%$ over the electron beam profile. This is confirmed by the excellent agreement between the fit in Figure 2C, which assumes no amplitude variation, and the data of Figure 2B.

\section{$\underline{\text { Resonator-free implementation }}$}

The mirror is a 70-nm thick aluminum layer and provides a good compromise between electron transmission $(\sim 30 \%)$ and $\mathrm{THz}$ reflectivity $(>90 \%)$. The p-polarized $\mathrm{THz}$ beam is incident at $60^{\circ}$ at the foil; the electron beam intersects at $27^{\circ}$. This angle combination ensures velocity matching over extended $\mathrm{THz}$ and electron beam (37). A single solenoid lens gently focuses the electron beam through the foil to a $55 \mu \mathrm{m}(\mathrm{rms})$ spot on the camera, located at a distance of $\sim 0.68 \mathrm{~m}$ behind the foil.

\section{Designing the electron-terahertz interaction}

An interaction between the electrons and terahertz which scales linearly with field is not possible in free space, because such an interaction (between one photon and one electron) cannot conserve momentum and energy simultaneously. Therefore, a third body must be used to engineer the interaction. The resonators used here localize the THz field to subwavelength dimensions so that the electron effectively passes through the region of strong fields faster than a THz period. Equivalently, the confinement of the electromagnetic field by the resonator leads to evanescent components which are phasematched to the electron (13). In the resonator-free implementation, the role of the third body is to abruptly truncate the electromagnetic fields so that the field seen by the electron changes abruptly in time $(36,37)$. The momentum change of the electron is given (to first order) by the integral of the Lorentz force on the electron's trajectory. The geometry of the interaction must be chosen to produce a momentum change in the desired control direction. Finally, the forces should be sufficiently uniform over the transverse profile of the beam.

Scaling of compression strength with electron energy

Here we briefly estimate how $\mathrm{THz}$ compression would work for other electron central energies than the $70 \mathrm{keV}$ in the experiment. For an electron moving along the $z$ axis with speed $v$ in a localized electromagnetic field, we can write the electron's energy increase in the longitudinal direction (ignoring quiver motion) as

$$
\delta W=q \int_{-\frac{l}{2}}^{\frac{l}{2}} E_{0}(z) e^{-i \omega\left(t(z)-t_{0}\right)} d z
$$

where $q$ is the charge of the electron, $t_{0}$ is a reference time, $t(z)=z / v$ is the time when the electron arrives at coordinate $z$, and $E_{0}$ is an envelope function describing the resonator mode, which decreases to zero at $\pm l / 2$. Here, it is assumed that the temporal envelope of the $\mathrm{THz}$ field changes slowly in comparison with the time that the electron interacts with the localized field. If the electron transit time through the fields is 
comparable to the $\mathrm{THz}$ period, then dependence on the electron velocity is non-trivial. In analogy with microwave acceleration, this expression can be formally rewritten as

$$
\delta W=q \delta V T e^{-i \omega t_{0}}
$$

where the static voltage change is

$$
\delta V \equiv \int_{-l / 2}^{l / 2} E_{0}(z) d z
$$

and the (dimensionless) transit-time factor is

$$
T \equiv \frac{\int_{-\frac{l}{2}}^{\frac{l}{2}} E_{0}(z) e^{-\frac{i \omega z}{v}} d z}{\int_{-l / 2}^{l / 2} E_{0}(z) d z}
$$

which accounts for the temporal variation of the electric field as the electron passes through the resonator. $T$ depends on a combination of $\mathrm{THz}$ frequency, field localization, and electron velocity and is generally not a monotonic function of these quantities. The maximum of the derivative of the longitudinal energy gain is thus

$$
g_{E}=\frac{d \delta W}{d t_{0}}=q \delta V \omega T
$$

In a simplified model, we can take the electric field amplitude to be uniform along $z$ and localized to the thickness of the resonator, which we now take as $l$. In this case (assuming $\pi l / \beta c$ is much less than the THz period)

$$
g_{E} \approx q E_{0} l \omega .
$$

In this approximation, the compression strength $\mathrm{gE}$ is independent of the electron energy, and the focus position scales according to Eq. (1) in the main paper. Thus THz field strengths of similar order-of-magnitude as used in the experiment will be suitable for a wide range of experimental conditions in electron diffraction, microscopy or other beam applications.

\section{$\underline{\mathrm{THz}}$ field strength calibration}

The THz field strength for the compression stage was calibrated approximately by measuring the THz power directly behind the silicon prism, for pump power from 1-5 W. To convert to a field strength, we used the field profile measured via electro-optic sampling and the focal parameters of the system. Independently, we measured deflection of the electron beam by the compression stage as a function of pump power used to generate the $\mathrm{THz}$, finding a linear relation between pump power and deflection in the range 3-7 W. The $\mathrm{THz}$ field strength for the streaking stage was estimated using electrooptic sampling measurements (25) with similar path lengths and focusing as in the experiment. 
Fitting the pulse duration of the uncompressed pulses

The pulse duration of the uncompressed pulses was determined from the deflectograms using a fitting routine which simultaneously determines pulse duration and the streaking field. The electron pulse profile is assumed to be Gaussian, and the streaking field is parameterized by a varying number of spline points. The deflection is assumed to be the same at all points in the transverse profile of the electron beam. For every set of parameters, a deflectogram can be calculated which includes the effect of streaking and convolution with the unstreaked beam profile at the camera. The error is then given by the rms difference between the measured and calculated deflectograms. The number of spline points is changed to verify that the fit is robust, and the quoted error values correspond to the interval where the error increases by $10 \%$ relative to the error of the optimal fit.

Streaking characterization of the compressed pulses

When the pulse duration is much less than the half period of the streaking terahertz (i.e., with the temporally compressed pulses), it is possible to make a direct reconstruction of monotonic segments of the streaking field, and using the streaking field, the temporal profiles of the electron pulses can be found without making assumptions about their shape.

If the pulses are sufficiently short, then the streaking field at the zero crossing is to a very good approximation linear over the range required to convert the streaking profile to a time-trace. This is illustrated in Figure S1, which shows the center of the streaked pulse vs. delay time. The linear calibration taken from this dataset was used to determine the time-axis in Figure 4D.

This linear calibration fails for longer pulses because the curvature of the streaking field vs. time distorts the streaked pattern, even when the pulse is streaked at the zero crossing. In this case, the streaking field reconstruction becomes important.

To reconstruct the streaking field from the deflectograms, we measure deflectograms with optimally compressed pulses, using a delay step which is much smaller than the electron pulse duration. We then average the deflectogram horizontally over a monotonic section of the deflectogram (Figure S1-A). This generates a "streaking profile" (Figure S1-B) that is equivalent to streaking a nearly rectangular pulse matched to this monotonic segment. The streaking profile corresponds to a number of electrons $\rho_{i}$ detected per pixel $i$. The central arrival time corresponding to deflection into pixel $i$ is $t_{i}=\sum_{n=1}^{i} \rho_{n} / I+t_{0}$, where $t_{0}$ is an arbitrary temporal offset, and $I$ is the effective number of electrons per unit time. The reconstructed deflection vs. time-delay (Figure $\mathrm{S} 1-\mathrm{C}$ ) is used to nonlinearly rescale the streaked pulses and determine the temporal profile of the electron pulses in Figure 3b. Due to the non-zero (unstreaked) beam profile on the camera, the streaking resolution is reduced at the turning points, which introduces distortion at the edges of the inferred temporal profiles of the longest pulses in Figure 3B. Note that the reconstruction in Fig. S1-C is truncated well before the turning points.

\section{Particle tracing simulations}

Simulations of pulse compression were carried out using General Particle Tracer (32). The distances between optical elements match the experimental distances within uncertainties of $\sim 5 \mathrm{~mm}$. The initial pulse duration at the cathode was $500 \mathrm{fs}$. The 
momentum distribution at the source was assumed to be hemispherical (41), and the energy spread was chosen to be $0.6 \mathrm{eV}$, to match the uncompressed pulse durations measured at the position of the streaking resonator. The rms source size of $5.3 \mu \mathrm{m}$ was chosen to reproduce the measured spot size at the compression point. The compression element was modeled as a thin scattering plane placed at $45^{\circ}$ to the beam and which imparts a momentum to the electrons based on their arrival time. The momentum change was oriented along the direction of maximum electric field in the resonator. It was assumed to be sinusoidal in time, uniform and synchronous across the plane of the resonator. The traces in Fig. 3B are histograms of the arrival time of $10^{4}$ particles at the streaking point. Aperturing of the beam was not taken into account, but in the experiment, the aperture is located at a point where the transverse spatial coordinate should not be correlated with arrival time. To simulate 3 fs (FWHM) pulses, the electron energy was increased to $150 \mathrm{keV}$, the initial energy spread was reduced to $0.2 \mathrm{eV}$, and the source size was reduced to $0.1 \mu \mathrm{m}(33)$. 

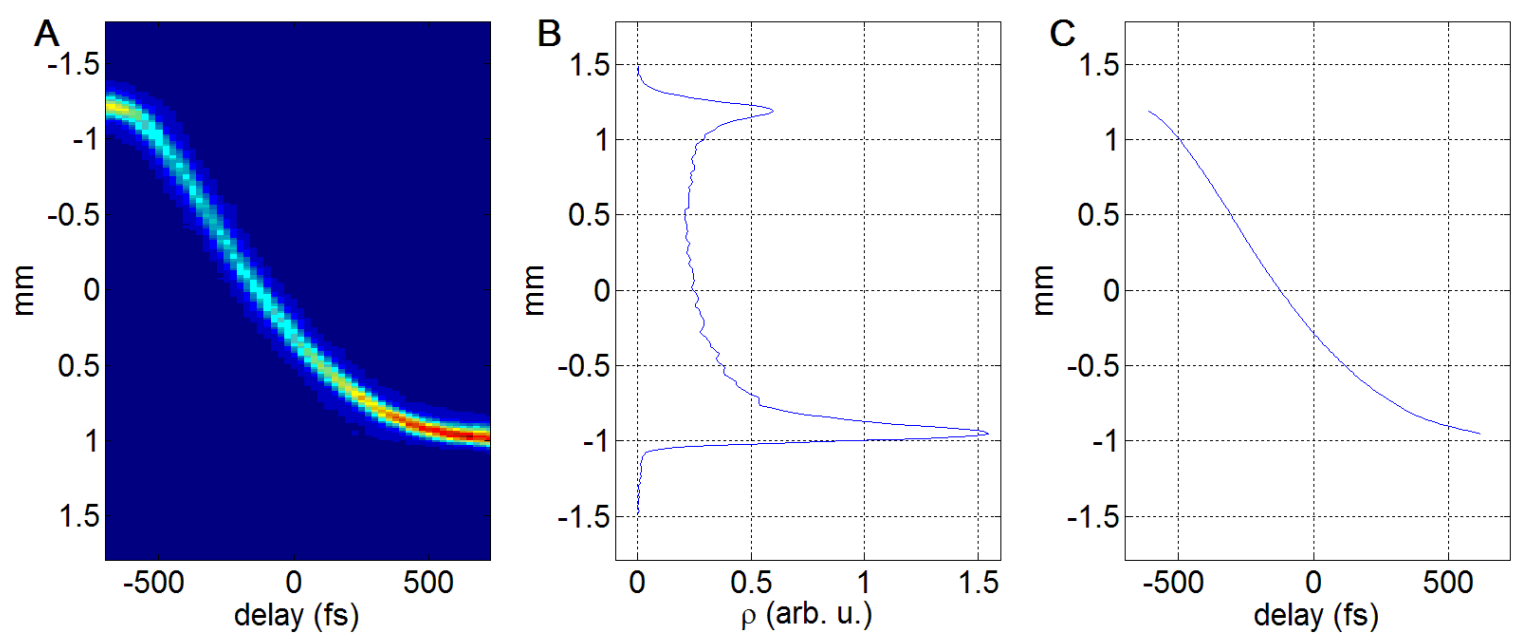

Fig. S1.

(A) Monotonic portion of a deflectogram used to infer streaking field. (B) Deflectograms as in (A) are integrated horizontally to obtain $\rho$ (several deflectograms are averaged for better signal-to-noise). (C) Numerically integrating $\rho$ results in the streaking field vs. time. 